\title{
Typologie des élevages de cerfs en Nouvelle-Calédonie et impact d'un suivi des performances d'abattage
}

\author{
S. Le Bel ${ }^{1}$ M. Salas ${ }^{1}$ D. Dulieu ${ }^{1}$
}

\begin{abstract}
Mots-clés
Cervidae - Cervus timorensis russa Cerf rusa - Classification - Abattage d'animaux - Carcasse - Pesée Nouvelle-Calédonie.
\end{abstract}

\begin{abstract}
Résumé
Une typologie des élevages de cerfs a été réalisée grâce à la méthode graphique de Bertin. Quatre grands types d'élevage qui se différenciaient essentiellement selon la superficie des pâturages, le chargement moyen et l'intensification des pratiques d'élevage ont pu être distingués. La campagne d'abattage de 1994 totalisait 1053 têtes dont 927 ont été concernées par le suivi. Les cerfs abattus étaient des mâles entiers (93 p. 100). Trente-cinq pour cent de l'effectif étaient âgés entre 1 et 2 ans et 48 p. 100 avaient plus de 2 ans. Le pourcentage de carcasses déclassées était de 1,6 p. 100 chez les éleveurs ayant opté pour la pesée comme outil de tri ; chez les autres éleveurs, il était sept fois plus important (12 p. 100 ; différence significative $p<0,001)$. Le poids vif $(P V)$ moyen des cerfs abattus $(n=510)$ était de $49,0 \pm 0,6 \mathrm{~kg}$, le poids carcasse $(P C)$ moyen $(n=927)$ à chaud après égouttage était de 28,4 $\pm 0,3 \mathrm{~kg}$ et le rendement à chaud (RD) moyen était de $56,8 \pm 0,3$ p. 100. Des analyses de la variance conduites sur les variables PV, PC et RD montrent que les facteurs types d'élevage, âge et saison d'abattage avaient un effet significatif. L'effet type d'élevage ne permet pas de discriminer un type d'exploitation plus performant. Les élevages extensifs (type 4) avaient des performances plus faibles par manque de finition de leurs animaux. De 1 à 2 ans, le PC a augmenté de $6 \mathrm{~kg}(25 \mathrm{~kg} / 31 \mathrm{~kg})$ mais le RD a diminué de 2 p. 100, de 58 à 56 p. 100. La meilleure saison d'abattage était la saison fraîche avec des carcasses de $30 \mathrm{~kg}$ et un rendement de $57 \mathrm{p} .100$. Une équation de régression significative $(p<0,001)$ permet de prédire le PC en fonction de PV $[P C(k g)=0,5 \mathrm{PV}(\mathrm{kg})+3,4]$. Vis-à-vis des autres pays éleveurs de cerfs rusa, les performances d'abattage obtenues en Nouvelle-Calédonie étaient moins bonnes. Cette différence de niveau de performance est à rattacher au mode d'élevage plus extensif du cerf rusa en Nouvelle-Calédonie. L'amélioration du niveau de production semble liée à une meilleure stratégie alimentaire pendant la saison sèche et à la finition des jeunes mâles abattus avant deux ans.
\end{abstract}

\section{INTRODUCTION}

L'élevage du cerf rusa (Cervus timorensis russa) en NouvelleCalédonie est une activité récente qui s'appuie sur une association d'éleveurs, l'EDEC*, regroupant un cheptel d'environ 10000 animaux. On compte environ une quinzaine d'élevages en production sur le Territoire. L'OCEF**, organisme territorial, se charge de l'abattage des animaux et de la commercialisation de la venaison.

1. CIRAD-EMVT, BP 186, Nouméa, Nouvelle-Calédonie

* EDEC : Etablissement de l'élevage de cervidés de Nouvelle-Calédonie

** OCEF : Office de commercialisation et d'entreposage frigorifique

$* * * 1 \mathrm{FCFP}=0,05 \mathrm{FF}$
Les pratiques d'élevage en vigueur sont sommaires et de type extensif : manipulations occasionnelles des animaux, peu ou pas d'interventions pendant le rut, sevrage et embouche exceptionnels, exploitation des parcours en rotation lente ou en pâturage continu (8). Le niveau de chargement moyen (3 cerfs/ha) est faible mais correspond à des pâturages de qualité moyenne à médiocre (18).

Le marché local de venaison assure l'écoulement de 1000 carcasses par an au rythme de 40 animaux tous les 15 jours. L'éleveur est rémunéré en fonction du poids carcasse à chaud selon deux classes : une classe premier choix correspondant à un poids carcasse de 23 à $45 \mathrm{~kg}$ (555 FCFP/kg***) et une classe de deuxième choix correspondant à un poids carcasse inférieur à 23 ou supérieur à $45 \mathrm{~kg}(250 \mathrm{FCFP} / \mathrm{kg})$. 
L'enquête structurelle menée en 1993 (8) identifia un certain nombre de contraintes au développement de la filière, notamment une fourniture irrégulière d'animaux de la part des éleveurs et l'hétérogénéité des carcasses produites.

Pour améliorer cette situation, un suivi des abattages fut proposé aux éleveurs de cerfs pour la campagne de 1994, avec notamment la pesée systématique des animaux destinés à être abattus.

Le but de la présente étude est de proposer une première classification des élevages de cerfs du Territoire et d'analyser l'impact d'un suivi des abattages dans les différents types d'élevage identifiés.

\section{MATERIEL ET METHODES}

\section{Lieu d'étude}

La Nouvelle-Calédonie représente $19000 \mathrm{~km}^{2}$ de terres émergées, à proximité du tropique du Capricorne dans la zone intertropicale. Le tiers de cette superficie est recouvert de savane et de formations herbeuses qui représentent l'espace pastoral.

Les élevages de cerfs sont localisés pour la plupart sur la façade Ouest de la Grande Terre (8).

Sur le plan climatique, l'année calendaire est découpée en trois saisons d'environ quatre mois : une saison des pluies (SP) chaude et humide de janvier à avril, une saison fraîche (SF) de mai à août et une saison sèche $(\mathrm{SS})$ de septembre à décembre.

\section{Typologie des élevages}

Les enquêtes ont porté sur 15 élevages. Parmi eux, un seul était situé sur la côte Est. Chaque élevage de cerfs a été caractérisé par une série de facteurs qualitatifs et quantitatifs décrivant le milieu, les caractéristiques structurelles de l'élevage, le cheptel et les principales pratiques mises en œuvre par l'éleveur. Afin d'effectuer un regroupement des 15 exploitations, une typologie utilisant la méthode graphique de Bertin (1) a été réalisée en utilisant une liste de facteurs discriminants « à dire d'expert » (tableau I) établie suite à une enquête préalable (8).

\section{Suivi des abattages}

La campagne d'abattage précédant l'étude (1993) s'est traduite par l'abattage de 1038 cerfs pour une production de 26,4 tonnes de venaison (valeur marchande de 11,2 millions FCFP). Le poids carcasse moyen était de 25,4 $\pm 0,5$ (15) et le pourcentage d'animaux déclassés pour poids insuffisant $(<$ à $23 \mathrm{~kg}$ ) était de 39 p. 100 (407/1 038).

En 1994, les abattages de cerfs ont été réalisés toute l'année sur un rythme bimensuel par lots de 40 têtes. Chez l'éleveur, les cerfs destinés à l'abattoir étaient pesés et triés en estimant le poids carcasse sur la base d'un rendement carcasse moyen de 58 p. 100 (9). Les animaux dont les poids carcasses étaient inférieurs à $23 \mathrm{ou} \mathrm{su}$ périeurs à $45 \mathrm{~kg}$ ont été écartés. Chez les éleveurs où les pesées n'ont pu être réalisées, un tri a été effectué sur des critères visuels. Une fois constitué, le lot de 40 cerfs était acheminé en bétaillère le jour même à l'abattoir de Bourail.

L'abattage des cerfs s'est effectué selon le procédé classiquement en usage dans les abattoirs de norme CEE (6). Un agent de l'OCEF a classé les carcasses en tenant compte de la dentition et du poids carcasse à chaud selon trois catégories. Chaque cerf abattu a été caractérisé par une série de facteurs qualitatifs et quantitatifs :
- le type d'élevage défini dont il était issu ;

- la période d'abattage (chaude, fraîche et sèche) ;

- le sexe ;

- l'âge présumé déterminé par l'état de la dentition (2,3), soit trois catégories : moins d'un an, de 1 à 2 ans, plus de deux ans (encadré 1) ;

- la catégorie OCEF (extra, classe 1 et classe 2);

- le poids vif avant abattage (PV en $\mathrm{kg}$ ) ;

- le poids carcasse à chaud (PC en $\mathrm{kg}$ ) ;

- le rendement à chaud (RD : 100 x PC/PV en p. 100).

\section{Analyses statistiques}

L'effet de la pesée sur le pourcentage de carcasses déclassées a été mesuré par le test du $\chi^{2}$ en comparant les taux de carcasses en seconde catégorie.

La même analyse a été utilisée pour apprécier la répartition des effectifs selon la période d'abattage, l'âge et le sexe des animaux, ainsi que les catégories OCEF.

L'homogénéité des variances a été testée avec le test de Lilliefors et Levene (procédure Examine SPSS/PC + version 5.0 (17)). Les effets des facteurs, types d'élevage, âges des animaux et période d'abattage sur les différentes variables prises en considération (PV, PC, RD) ont été estimés par des analyses de variance (procédure Anova SPSS/PC + version 5.0 (17)). Pour la variable RD exprimée en pourcentage, l'analyse de variance a été réalisée sur l'arcsinus des valeurs obtenues. L'estimation du poids carcasse à partir du poids vif a été effectuée par le biais d'une régression entre les poids carcasses et les poids vifs (procédure Regression SPSS/PC + version 5.0 (17)).

\section{RESU LTATS}

\section{Typologie des élevages de cerfs}

Le traitement graphique de l'information par l'outil de Bertin est résumé dans le tableau II. Le facteur « Capture de cerfs sauvages » (tableau I) n'a pas été retenu dans la mesure où tous les élevages, à l'exception d'un seul (le $\left.n^{\circ} 15\right)$, avaient la possibilité d'effectuer des captures.

\section{Encadré 1}

Méthode de classification des carcasses de cerfs à l'abattoir (source OCEF)

\begin{tabular}{|c|c|c|c|}
\hline Catégories & Extra & Première & Seconde \\
\hline Dentition & $|-0|-1$, & I-2 à |-4 & I-0 à I-4 \\
\hline $\begin{array}{l}\text { Poids carcasse } \\
(\mathrm{kg})\end{array}$ & $23-45$ & $23-45$ & $<23$ ou $>45$ \\
\hline $\begin{array}{l}\text { Prix achat OCEF } \\
\text { (FCFP } / \mathrm{kg})\end{array}$ & 555 & 555 & 255 \\
\hline
\end{tabular}

I-0 : absence d'incisive de remplacement

I-1 : présence des pinces de remplacement

I-2: présence des pinces et des premières mitoyennes de remplacement I-3 : présence des pinces, des premières et deuxièmes mitoyennes de remplacement

1-4 : présence des pinces, des mitoyennes et des coins de remplacement La classe extra et première sont payées au même tarif 
Tableau I

Facteurs discriminants « à dire d'expert » retenus pour l'élaboration de la typologie

\begin{tabular}{|c|c|c|c|c|}
\hline \multicolumn{4}{|c|}{ Correspondances graphiques avec le tableau II } & \\
\hline Caractéristiques du milieu & $\begin{array}{l}\text { Pluviométrie }(\mathrm{mm}) \\
\text { Cours d'eau } \\
\text { Surface agricole utilisable (ha) } \\
\% \text { mécanisable }\end{array}$ & $\begin{array}{l}<800 \\
\text { Absent } \\
>200 \\
25-50\end{array}$ & $\begin{array}{c}800-1500 \\
- \\
100-200 \\
50-75\end{array}$ & $\begin{array}{l}>1500 \\
\text { Permanent } \\
\quad<100 \\
>75\end{array}$ \\
\hline G estion du milieu & $\begin{array}{l}\text { SAU } / \mathrm{km} \text { de barrière }(\mathrm{ha} / \mathrm{km}) \\
\% \text { amélioré } \\
\text { Arbustes fourragers } \\
\text { Gyrobroyage } \\
\text { Rotation }\end{array}$ & $\begin{array}{l}>20 \\
<10 \\
\text { Absents } \\
\text { N on } \\
\text { N on }\end{array}$ & $\begin{array}{c}10-20 \\
10-30 \\
- \\
- \\
-\end{array}$ & $\begin{array}{c}<10 \\
>30 \\
\text { Présents } \\
\text { O ui } \\
\text { O ui }\end{array}$ \\
\hline Cheptel & $\begin{array}{l}\text { Effectif de cervidés } \\
\text { Densité (cerfs/ha) }\end{array}$ & $\begin{array}{c}>500 \\
<2\end{array}$ & $\begin{array}{c}200-500 \\
2-5\end{array}$ & $\begin{array}{c}<200 \\
>5\end{array}$ \\
\hline G estion du cheptel & $\begin{array}{l}\text { Capture de cerfs sauvages } \\
\text { Cheptel identifié } \\
\text { Manipulations } \\
\text { Sevrage et embouche } \\
\text { Agrégation des troupeaux }\end{array}$ & $\begin{array}{l}\text { O ui } \\
\text { Non } \\
1 \text { fois/an } \\
\quad \text { Non } \\
1 \text { troupeau }\end{array}$ & $\begin{array}{l}\text { - } \\
\text { Partiellement } \\
2 \text { à } 3 \text { fois/an } \\
\text { Partiellement }\end{array}$ & $\begin{array}{c}\text { Non } \\
\text { Totalement } \\
>3 \text { fois/an } \\
\text { O ui } \\
2 \text { troupeaux }\end{array}$ \\
\hline
\end{tabular}

Tableau II

Typologie des élevages de cerfs en Nouvelle-Calédonie

\begin{tabular}{|c|c|c|c|c|c|c|c|c|c|c|c|c|c|c|c|}
\hline Types d'élevage & \multicolumn{3}{|c|}{ Type 1} & \multicolumn{4}{|c|}{ Type 2} & \multicolumn{4}{|c|}{ Type 3} & \multicolumn{3}{|c|}{ Type 4} & Autre \\
\hline Eleveurs : & 15 & 9 & 4 & 1 & 7 & 5 & 6 & 11 & 8 & 14 & 2 & 13 & 3 & 10 & 12 \\
\hline Gyrobroyage & & & & & & & & & & & & & & & \\
\hline Manipulations & & & & & & & & & & & & & & & \\
\hline Cheptel identifié & & & & & & & & & & & & & & & \\
\hline $\begin{array}{l}\text { A grégation } \\
\text { troupeaux }\end{array}$ & & & & & & & & & & & & & & & \\
\hline$\%$ mécanisable & & & & & & & & & & & & & & & \\
\hline Rotation & & & & & & & & & & & & & & & \\
\hline $\mathrm{SAU} / \mathrm{km}$ de barrière & & & & & & & & & & & & & & & \\
\hline$\%$ amélioré & & & & & & & & & & & & & & & \\
\hline Densité cerfs/ha & & & & & & & & & & & & & & & \\
\hline $\begin{array}{l}\text { Sevrage et } \\
\text { embouche }\end{array}$ & & & & & & & & & & & & & & & \\
\hline Cours d'eau & & & & & & & & & & & & & & & \\
\hline SAU & & & & & & & & & & & & & & & \\
\hline Effectif de cervidés & & & & & & & & & & & & & & & \\
\hline Arbustes fourragers & & & & & & & & & & & & & & & \\
\hline Pluviométrie & & & & & & & & & & & & & & & \\
\hline
\end{tabular}

Voir tableau I pour correspondances graphiques

Il a été possible de distinguer quatre types d'élevage, du système le plus intensif au plus extensif.

L'élevage $n^{\circ} 12$ était atypique en raison de sa localisation géographique sur la façade Est de la Grande Terre avec une pluviométrie moyenne de 2000 à $3000 \mathrm{~mm}$ d'eau par an. Etant donné le mode de gestion en vigueur dans cet élevage et la pauvreté des ressources fourragères, cet élevage a été rattaché à ceux de type 4 .

Type $1(n=3)$

Les élevages de faible superficie au mode de gestion intensif ont été regroupés. Les principales caractéristiques étaient les suivantes :
- la surface agricole utilisée (SAU), inférieure à 100 ha, était en grande partie mécanisable. Le facteur précipitation n'était pas limitant, la pluviométrie étant supérieure à $800 \mathrm{~mm}$ ou l'éleveur disposant d'un système d'irrigation ;

- le cheptel, de moins de 500 têtes, provenait de captures sauf dans le cas de l'élevage ${ }^{\circ} 15$. Le chargement moyen était supérieur à 5 cerfs/ha ;

- plus de 30 p. 100 de la SAU était améliorée. L'importance du cloisonnement a permis d'exploiter le pâturage en rotation. Les refus étaient gyrobroyés régulièrement ; 
- avec 100 p. 100 d'individus identifiés, les animaux répartis en deux troupeaux (commercial et reproduction) étaient régulièrement manipulés, notamment à l'occasion d'opérations de sevrage et d'embouche.

\section{Type $2(n=4)$}

Les élevages se rapprochant du type 1 ont été regroupés mais avec un niveau d'intensification moins important. La SAU était de 100 à 200 ha et la taille du cheptel était comparable à celle du type 1 . Ces élevages se distinguaient entre autres par un niveau de chargement ( 2 à 5 cerfs/ha) et d'amélioration fourragère plus faible (10 à 30 p. $100 \mathrm{SAU})$.

Type $3(n=4)$

Les élevages extensifs ayant partiellement amélioré leur SAU ont été regroupés. Ils se sont caractérisés par :

- une SAU souvent supérieure à 200 ha dont le pourcentage mécanisable était limité à 30 p. 100. L'irrigation de certaines parcelles a permis de compenser partiellement les effets d'une pluviométrie irrégulière et inférieure à $800 \mathrm{~mm}$;

- un cheptel d'au moins 500 têtes provenait de captures. Le chargement moyen était de 2 à 5 cerfs/ha ;

- moins de 10 p. 100 de la SAU était améliorée. L'exploitation du parcours s'est effectuée en pâturage continu. Le gyrobroyage des adventices était une pratique courante ;

- les cerfs, en partie identifiés, ont été regroupés en un seul troupeau. Les manipulations s'effectuaient à l'occasion d'opérations commerciales. Il n'y avait pas d'embouche et les sevrages étaient occasionnels.

\section{Type $4(n=3)$}

Les élevages de grande superficie au mode de gestion extensif ont été regroupés. Les principales caractéristiques étaient les suivantes :

- la SAU de plus de 500 ha était partiellement mécanisable (30 p. 100 de la SAU). A l'absence de système d'irrigation s'est ajoutée une pluviométrie inférieure à $800 \mathrm{~mm}$;

- le cheptel, acquis par capture, s'élevait au minimum à 500 têtes. Le niveau de chargement était faible avec souvent moins de 2 cerfs/ha ;

- avec moins de 10 p. 100 de la SAU améliorée, l'exploitation des parcours s'est effectuée en continu. L'entretien des parcelles était quasi nul ;

- les cerfs, regroupés en un seul troupeau, étaient manipulés uniquement à l'occasion d'opérations commerciales.

\section{Caractéristiques des abattages selon les différents types d'élevage}

Au cours de la campagne d'abattage de 1994, 1053 cerfs ont été abattus. Les suivis d'abattage ont porté sur un échantillon de 927 cerfs, soit 88 p. 100 des animaux abattus. Les principaux résultats de ces abattages sont exposés dans le tableau III.

L'abattage des cerfs s'est effectué en 35 séquences par lots d'environ 26 cerfs (min./max. : 8 à 42). En se référant à la typologie des élevages, 11 p. 100 des cerfs abattus provenaient d'exploitations de type 1,22 p. 100 de celles de type 2, 40 p. 100 de celles de type 3 et 27 p. 100 de celles de type 4 .

\section{Tableau III}

Répartition des effectifs abattus selon la typologie de l'élevage, la période d'abattage, le sexe, l'âge de l'animal, les catégories O CEF et la pratique de la pesée

\begin{tabular}{|c|c|c|c|c|c|c|}
\hline \multirow[b]{2}{*}{$\begin{array}{l}\text { Effectif abattus } \\
\text { (\% effectif total) }\end{array}$} & & Ensemble des élevages & $\begin{array}{l}\text { Elevages } \\
\text { de type } 1\end{array}$ & $\begin{array}{l}\text { Elevages } \\
\text { de type } 2\end{array}$ & $\begin{array}{r}\text { Elevages } \\
\text { de type } 3\end{array}$ & $\begin{array}{r}\text { Elevages } \\
\text { de type } 4\end{array}$ \\
\hline & & $\begin{array}{c}927 \\
(100 \%)\end{array}$ & $\begin{array}{c}106 \\
(11 \%)\end{array}$ & $\begin{array}{c}200 \\
(22 \%)\end{array}$ & $\begin{array}{c}374 \\
(40 \%)\end{array}$ & $\begin{array}{c}247 \\
(27 \%)\end{array}$ \\
\hline \multirow[t]{3}{*}{ Période d'abattage (a) } & Saison des pluies & $255(28 \%)$ & 0 & 40 & 80 & 135 \\
\hline & Saison fraîche & $380(41 \%)$ & 84 & 100 & 196 & 0 \\
\hline & Saison sèche & $292(32 \%)$ & 22 & 60 & 98 & 112 \\
\hline \multirow[t]{2}{*}{ Sexe (b) } & Mâle & $858(93 \%)$ & 97 & 200 & 315 & 246 \\
\hline & Femelle & $69(7 \%)$ & 9 & 0 & 59 & 1 \\
\hline \multirow[t]{3}{*}{ Age d'abattage (c) } & Moins d'un an & $153(17 \%)$ & 45 & 43 & 44 & 21 \\
\hline & De 1 à 2 ans & $325(35 \%)$ & 30 & 62 & 149 & 84 \\
\hline & Plus de 2 ans & $449(48 \%)$ & 31 & 95 & 181 & 142 \\
\hline \multirow[t]{2}{*}{ Catégories O CEF (d) } & Extra, première & $871(94 \%)$ & 104 & 190 & 350 & 227 \\
\hline & Seconde & $56(6 \%)$ & 2 & 10 & 24 & 20 \\
\hline \multirow[t]{2}{*}{ Pratique de la pesée (e) } & O ui & $510(55 \%)$ & 106 & 59 & 228 & 117 \\
\hline & Non & $417(45 \%)$ & 0 & 141 & 146 & 130 \\
\hline
\end{tabular}

$a, b, c, e$ : répartition significativement différente selon les types d'élevages $\left(\chi^{2}, p<0,001\right)$

$\mathrm{d}$ : pas de différence significative $\left(\chi^{2}, \mathrm{p}>0,05\right)$ 
Vingt-huit pour cent des abattages ont été effectués en saison chaude, 41 p. 100 en saison fraîche et 32 p. 100 en saison sèche. La répartition des abattages par saison diffère significativement selon le type d'élevage ( $\mathrm{p}<0,001)$. Si les types 1,2 et 3 ont abattu 50 à 72 p. 100 de leur cheptel en saison fraîche, les élevages extensifs de type 4 n'ont réalisé aucun abattage pendant cette période.

Les cerfs abattus étaient à 93 p. 100 des mâles entiers $(n=858)$ et à 7 p. 100 des biches $(n=69)$. La proportion de mâles ou de femelles abattus selon le type d'élevage est significativement différente $(\mathrm{p}<0,001)$; les élevages de type 3 ont ainsi abattu proportionnellement le plus de femelles, soit 16 p. 100 de leur effectif (59/374)

En se basant sur la dentition des individus, l'effectif abattu était constitué pour moitié (48 p. 100) d'animaux âgés d'au moins deux ans et pour un tiers de cerfs de 1 à 2 ans (35 p. 100). La part des individus de moins d'un an ne représentait que 17 p. 100 de l'effectif. Selon le type d'élevage, cette répartition diffère significativement $(p<0,001)$. Dans les élevages de type intensif (type 1$)$, la proportion d'animaux abattus de moins d'un an représentait 42 p. 100 de l'effectif (45/106). Ce pourcentage a diminué progressivement chez les autres types d'élevage pour atteindre $9 \mathrm{p}$. 100 de l'effectif dans les élevages extensifs (type 4).

En se référant à la grille de classification OCEF, les carcasses classées en classe extra ou première représentaient 94 p. 100 de l'effectif, le reste étant des carcasses déclassées en seconde catégorie (6 p. 100). La proportion de carcasses classées en catégorie première ou extra n'est pas significativement différente selon les types d'élevage.

\section{Suivi des abattages}

\section{Résultats globaux}

Cinquante-cinq pour cent des cerfs suivis dans cet étude ont été pesés et triés (tableau III), selon le protocole proposé avant d'être abattus. Le reste de l'effectif (45 p. 100) a été abattu sans pesée préalable. Selon le type d'élevage, le suivi a concerné une proportion plus ou moins importante d'animaux, la répartition n'étant pas homogène selon le type d'élevage ( $\mathrm{p}$ < 0,001). Ainsi 100 p. $100 \mathrm{du}$ cheptel a été pesé dans les élevages de type 1,30 p. 100 pour le type 2, 61 p. 100 pour le type 3 et 47 p. 100 pour le type 4 .

Pour les élevages qui ont opté pour la pesée et le tri des cerfs avant abattage, le taux de carcasses classées en seconde catégorie était de 1,6 p. 100. Chez les éleveur qui n'ont pas adhéré pas au suivi il était sept fois plus élevé (12 p. 100), la différence observée étant significative $(\mathrm{p}<0,001)$.

Le poids vif moyen des cerfs abattus était de 49,0 $\pm 0,6 \mathrm{~kg}$ pour une fourchette min./max. de 38,1 à $76,0 \mathrm{~kg}$. Le poids carcasse moyen à chaud après égouttage était de $28,4 \pm 0,3 \mathrm{~kg}$ pour une fourchette min./max. de 18,5 à $53,5 \mathrm{~kg}$. Le calcul du rendement à chaud donne un rendement moyen de $56,8 \pm 0,3$ p. 100 pour un écart min./max. de 43,0 à 65,5 p. 100. La campagne d'abattage de 1994 a représenté un tonnage de 29,9 tonnes pour une valeur marchande de 15,9 millions FCFP.

\section{Analyse des facteurs de variations}

L'analyse de la variance, lors de tests de normalité, a présenté une bonne robustesse en ce qui concerne la non-normalité lorsque les effectifs étaient suffisamment grands $(5,17)$.
Compte tenu de la faiblesse de l'effectif de biches abattues $(n=69)$ et surtout des biches pesées avant abattage $(n=7)$, l'effet du facteur sexe n'a pas été analysé. On note seulement un poids carcasse moyen significativement plus faible par rapport aux mâles (respectivement $28,8 \pm 0,8$ et $28,1 \pm 0,5 \mathrm{~kg}, \mathrm{p}<0,001)$.

Afin d'analyser l'effet des facteurs type d'élevage, âge et période d'abattage sur les PV, PC et RD, des analyses de variance ont été effectuées avec les modalités suivantes : type d'élevage (types 1, 2, 3 et 4), âge (moins d'un an, un à deux ans, plus de deux ans), période d'abattage (saison des pluies, saison fraîche et saison sèche).

Pour la variable poids vif (tableau IV), les trois facteurs retenus ont un effet significatif avec un écart-type résiduel de $5,5 \mathrm{~kg}$ et un pourcentage de variance expliquée de 22 p. 100. Le calcul des moyennes ajustées montre que :

- la valeur du PV oscille de 45 à $53 \mathrm{~kg}$ selon le type d'élevage considéré, il est notamment du même ordre chez les élevages les plus intensifs (type $1, \mathrm{PV}=50,8 \mathrm{~kg}$ ) et les plus extensifs (type 4, $\mathrm{PV}=51,6 \mathrm{~kg})$;

- l'abattage de la classe 2 ans permet de travailler des animaux plus lourds (PV ajusté $53 \mathrm{~kg}$ ) avec néanmoins un gain pondéral limité à $8 \mathrm{~kg}$ par rapport à la classe d'âge de 1 an (PV ajusté $44 \mathrm{~kg}$ ) ;

- la saison fraîche est la période où les cerfs les plus lourds sont abattus avec une valeur de PV de $50 \mathrm{~kg}$.

Pour la variable poids carcasse (tableau V), les PV et PC étant liés, des résultats similaires avec un effet significatif des trois facteurs étudiés ont été observés (écart-type résiduel $4,4 \mathrm{~kg}$, pourcentage de variance expliquée 12 p. 100).

Pour la variable rendement à chaud (tableau VI), l'analyse de la variance après transformation de la variable montre que les trois facteurs ont un effet significatif avec un écart-type résiduel de 0,029 et un pourcentage de variance expliquée de 31 p. 100. Les meilleurs rendements d'abattage s'observent avec de jeunes cerfs d'un an (RD moyen 58 p. 100), provenant d'élevages de type 3 (RD moyen 59 p. 100) et abattus en saison fraîche (RD moyen 58 p. 100).

\section{Prédiction du poids carcasse à chaud}

Une régression a été réalisée sur les couples poids carcasses et poids vifs exprimés en $\mathrm{kg}(\mathrm{n}=510)$. La régression est significative et l'équation de prédiction du PV est la suivante :

$\mathrm{PC}(\mathrm{kg}): 0,5 \mathrm{PV}(\mathrm{kg})+3,4 ;\left(\mathrm{p}<0,001 ; \mathrm{r}^{2}=0,81\right.$; écart-type résiduel $=1,7 \mathrm{~kg})$.

\section{DISCUSSION}

Par rapport à 1993, le bilan de la campagne d'abattage de 1994 $(15,16)$ s'est notablement amélioré. En abattant un effectif équivalent d'un millier de cerfs (1 038 cerfs en 1993 et 1053 cerfs en 1994), on enregistre une augmentation du tonnage carcasse produit de 13,1 p. 100 ( $+3,5$ tonnes), du poids carcasse moyen de 12,6 p. 100 $(+3,2 \mathrm{~kg})$ et de la valeur marchande des abattages de 42 p. 100 (+ 4,7 millions FCFP), ainsi qu'une diminution du pourcentage de carcasses en classe 2 de 86 p. 100 (- 33,7 p. 100). Travaillant avec les mêmes éleveurs, dans un contexte climatique persistant de sécheresse $(13,14)$, la mise en pratique des pesées avant abattage s'est apparemment accompagnée d'une amélioration globale de la qualité des abattages. 


\section{Tableau IV}

Résultats de l'analyse de variance sur le poids vif avec le type d'élevage, l'âge et la période d'abattage comme facteurs

\begin{tabular}{lrrrrr} 
& Somme des carrés & Degré de liberté & Carré moyen & Valeur de F & Signification \\
\hline Typologie (1) & & 3 & 1554,5 & 51,3 & $\mathrm{p}<0,001$ \\
Période d'abattage (2) & 4663,6 & 2 & 94,3 & 3,1 & $\mathrm{p}=0,045$ \\
Age d'abattage (3) & 5800,7 & 2 & 2900,0 & 95,7 & $\mathrm{p}<0,001$ \\
Variance résiduelle & 15210,2 & 502 & 30,3 &
\end{tabular}

Calcul des moyennes ajustées (kg) selon le type d'élevage, l'âge et la période d'abattage (effectif)

$\begin{array}{lcccc}\text { Type d'élevage } & \text { Type 1 } & \text { Type 2 } & \text { Type 3 } & \text { Type 4 } \\ & 50,8(106) & 53,3(59) & 45,6(228) & 51,6(117) \\ \text { Période d'abattage } & \text { Saison des pluies } & \text { Saison fraîche } & \text { Saison sèche } & \\ & 47,9(108) & 49,7(272) & 48,2(130) & \\ \text { Age d'abattage } & \text { Moins d'un an } & \text { Un à deux ans } & \text { Plus de deux ans } & \\ & 44,1(113) & 48,0(216) & 53,2(181)\end{array}$

\section{Tableau V}

Résultats de l'analyse de variance sur le poids carcasse avec le type d'élevage, l'âge et la période d'abattage comme facteurs

\begin{tabular}{|c|c|c|c|c|c|}
\hline & Somme des carrés & Degré de liberté & Carré moyen & Valeur de F & Signification \\
\hline $\begin{array}{l}\text { Typologie (1) } \\
\text { Période d'abattage (2) } \\
\text { Age d'abattage (3) } \\
\text { Variance résiduelle }\end{array}$ & $\begin{array}{r}673,5 \\
1070,4 \\
4271,4 \\
17149,3\end{array}$ & $\begin{array}{r}3 \\
2 \\
2 \\
919\end{array}$ & $\begin{array}{r}224,7 \\
535,2 \\
2135,7 \\
18,7\end{array}$ & $\begin{array}{r}12,0 \\
28,7 \\
114,4\end{array}$ & $\begin{array}{l}p<0,001 \\
p<0,001 \\
p<0,001\end{array}$ \\
\hline \multicolumn{6}{|c|}{ Calcul des moyennes ajustées ( $\mathrm{kg}$ ) selon le type d'élevage, l'âge et la période d'abattage (effectif) } \\
\hline Type d'élevage & $\begin{array}{c}\text { Type } 1 \\
28,5(106)\end{array}$ & $\begin{array}{c}\text { Type } 2 \\
28,9(200)\end{array}$ & $\begin{array}{c}\text { Type } 3 \\
27,3(374)\end{array}$ & $\begin{array}{c}\text { Type } 4 \\
29,4(247)\end{array}$ & \\
\hline Période d'abattage & $\begin{array}{c}\text { Saison des pluies } \\
26,8(255)\end{array}$ & $\begin{array}{c}\text { Saison fraîche } \\
29,9(380)\end{array}$ & $\begin{array}{c}\text { Saison sèche } \\
27,8(292)\end{array}$ & & \\
\hline Age d'abattage & $\begin{array}{l}\text { Moins d'un an } \\
24,9 \text { (153) }\end{array}$ & $\begin{array}{l}\text { Un à deux ans } \\
27,0(325)\end{array}$ & $\begin{array}{c}\text { Plus de deux ans } \\
30,5(449)\end{array}$ & & \\
\hline
\end{tabular}

\section{Tableau VI}

Résultats de l'analyse de variance sur l'arcsinus de la racine carrée du rendement d'abattage (\%) avec le type d'élevage, l'âge et la période d'abattage comme facteurs

\begin{tabular}{|c|c|c|c|c|c|}
\hline & Somme des carrés & Degré de liberté & Carré moyen & Valeur de F & Signification \\
\hline $\begin{array}{l}\text { Typologie (1) } \\
\text { Période d'abattage (2) } \\
\text { Age d'abattage (3) } \\
\text { Variance résiduelle }\end{array}$ & $\begin{array}{l}0,120 \\
0,013 \\
0,021 \\
0,426\end{array}$ & $\begin{array}{r}3 \\
2 \\
2 \\
502\end{array}$ & $\begin{array}{l}0,040 \\
0,006 \\
0,010 \\
0,001\end{array}$ & $\begin{array}{r}47,0 \\
7,5 \\
12,2\end{array}$ & $\begin{array}{l}p<0,001 \\
p=0,001 \\
p<0,001\end{array}$ \\
\hline \multicolumn{6}{|c|}{ Calcul des rendements moyens (\% ) selon le type d'élevage, l'âge et la période d'abattage (SD , effectif) } \\
\hline Type d'élevage & $\begin{array}{c}\text { Type } 1 \\
56,8(3,9106)\end{array}$ & $\begin{array}{c}\text { Type } 2 \\
56,5(1,859)\end{array}$ & $\begin{array}{c}\text { Type } 3 \\
58,4(2,8228)\end{array}$ & $\begin{array}{c}\text { Type } 4 \\
53,8(3,0117)\end{array}$ & \\
\hline Période d'abattage & $\begin{array}{l}\text { Saison des pluies } \\
55,7(3,2108)\end{array}$ & $\begin{array}{l}\text { Saison fraîche } \\
57,8(3,1272)\end{array}$ & $\begin{array}{l}\text { Saison sèche } \\
55,5(3,8130)\end{array}$ & & \\
\hline Age d'abattage & $\begin{array}{c}\text { Moins d'un an } \\
57,9(2,9113)\end{array}$ & $\begin{array}{l}\text { Un à deux ans } \\
56,8(3,5216)\end{array}$ & $\begin{array}{c}\text { Plus de deux ans } \\
56,0(3,6181)\end{array}$ & & \\
\hline
\end{tabular}


L'analyse de l'effet pesée est à replacer dans le contexte de redynamisation de la filière cervine menée par l'EDEC avec l'appui du CIRAD en 1994 (11). L'effet pesée chez les éleveurs ayant opté pour cette pratique traduit à la fois une meilleure préparation des lots d'abattage et un effet filtre de la pesée. Cette hypothèse est étayée par la diminution du taux de carcasses déclassées en 1994 (39 p. 100 en 1993 et 12 p. 100 en 1994) chez les éleveurs participant à cette dynamique mais ne pesant pas leurs lots d'abattage.

Dans le cadre de ce suivi, les cerfs abattus pesaient en moyenne $49 \mathrm{~kg}$ et fournissaient avec un rendement moyen de 57 p. 100 des carcasses d'environ $28 \mathrm{~kg}$. Ces résultats concordent avec les observations menées par Chardonnet sur une trentaine de cerfs sauvages en Nouvelle-Calédonie (3). Hormis les résultats d'embouche menée en station expérimentale (9), les performances d'abattage obtenues en Nouvelle-Calédonie apparaissent en moyenne moins bonnes que celles observées dans les autres pays éleveurs de cerf rusa. A l'Ile Maurice, le poids carcasse moyen des animaux abattus à 2 ans est, d'après Chardonnet (4), de 32 à $34 \mathrm{~kg}$. En Australie, où les éleveurs abattent leurs daguets vers 24 mois, Woodford enregistre des poids carcasse de $42 \mathrm{~kg}$ avec un rendement de 59 p. $100(21,22)$.

Cette différence de niveau de performance est à rattacher au mode d'élevage plus extensif du cerf rusa en Nouvelle-Calédonie $(8,10)$. Sevré en début de saison sèche, le jeune faon bénéficie rarement d'une supplémentation adéquate. L'embouche lorsqu'elle est pratiquée, se limite à la pâture de réserves fourragères herbacées sur pieds. Dans ce contexte, la croissance du faon en post-sevrage est fortement ralentie, voire stoppée, pendant la saison sèche et ne bénéficie que d'un faible rattrapage en saison des pluies.

Des essais d'alimentation ad libitum menés sur des faons mâles en Nouvelle-Calédonie (7) et en Australie (20,22) confirment cette hypothèse en démontrant qu'avec un régime adéquat le profil de croissance du faon est linéaire et permet d'obtenir vers 14-16 mois des animaux pesant 80 à $93 \mathrm{~kg}$ et fournissant des carcasses de plus de $50 \mathrm{~kg}$.

Dans cet essai, la meilleure période d'abattage apparaît comme étant la saison fraîche avec un PV de $50 \mathrm{~kg}$, un PC de $30 \mathrm{~kg}$ et un rendement à chaud de 57 p. 100. Malgré un infléchissement de la production $(18,19)$, cette saison qui bénéficie d'une biomasse de meilleure qualité nutritionelle correspond à l'époque du rut où les animaux sont en bon état corporel (3).

En tablant sur un poids à la naissance de 3,5 kg (12), le PV du cerf rusa est multiplié par 10 la première année puis progresse de 20 p. 100 entre 1 et 2 ans. Woodford $(20,21)$, en travaillant sur le profil de la courbe de croissance, aboutit à la même conclusion en montrant que celle-ci s'infléchit nettement après 15 mois. L'évolution du rendement carcasse subit le même type d'évolution en diminuant dans cet essai de 58 à 56 p. 100 après 24 mois. Ces résultats soulignent l'intérêt d'abattre les jeunes mâles avant deux ans. Au-delà de cet âge, les éleveurs seront doublement pénalisés par l'entretien d'un cheptel pendant une seconde saison sèche et l'élevage d'animaux dont les performances de croissance sont faibles.

La typologie obtenue au cours de ce travail a une valeur relativement limitée. En effet, l'échantillon de fermes enquêtées est faible $(n=15)$ et la plupart des fermes sont très récentes ou en phase de démarrage. Il existe donc une relative homogénéité des situations. Il n'y a pas eu de laps de temps assez long pour permettre aux fermes de se distinguer par des trajectoires d'évolution différentes. Cette typologie est un outil de travail provisoire qui devra être affiné avec le temps. En considérant les quatre types d'élevage, du plus intensif (type 1) au plus extensif (type 4), il n'y a apparem- ment pas d'effet marquant de la typologie sur les résultats d'abattage, sauf en ce qui concerne le rendement à chaud plus faible chez les élevages extensifs du groupe 4. La pratique de la pesée semble avoir agi comme un filtre en éliminant les sujets les moins conformés. L'effet filtre est apparu aux auteurs plus fort dans les élevages les moins bien conduits. Cette hypothèse reste néanmoins à confirmer en l'absence d'appréciation du taux de rejet selon les élevages.

En intégrant l'âge des animaux abattus, il est toutefois possible de distinguer deux stratégies de production : celle des élevages intensifs (type 1) avec abattage précoce des jeunes mâles plus ou moins finis et celle des élevages extensifs (type 4) où l'abattage concerne le tout-venant avec une forte proportion de cerfs adultes. L'étape suivante serait d'apprécier la rentabilité économique de ces deux systèmes pour savoir lequel des deux est le mieux adapté au contexte néo-calédonien.

Vis-à-vis des autres pays éleveurs de cerfs rusa, la NouvelleCalédonie dispose d'une certaine marge de progression sur le plan qualitatif et quantitatif. Parmi les voies d'amélioration qui méritent d'être explorées, la première serait d'inciter les éleveurs à soigner la finition des daguets en visant un poids carcasse de $30 \mathrm{~kg}$ et un rendement carcasse de 60 p. 100.

\section{BIBLIO GRAPHIE}

1. BERTIN J., 1977. La graphique et le traitement graphique de I'information. Paris, France, Flammarion, 273 p.

2. BIANCHI M., 1993. Reproduction du cerf rusa de java (Cervus timorensis russa) en N ouvelle-Calédonie. N ouméa, N ouvelle-Calédonie, CIRAD-EM VT, 41 p. (Rapport Cordet-M ED ETO M)

3. CHARDONNET P., 1988. Etude de faisabilité technique et économique de l'élevage de cerfs en Nouvelle-Calédonie. MaisonsAlfort, France, CIRAD-IEM VT, $282 \mathrm{p}$.

4. CHARD O N NET P., 1988. O bservations et réflexions sur l'élevage de cerfs à I'Ile Maurice. Maisons-Alfort, France, CIRAD-IEMVT, $42 \mathrm{p}$. (Rapport IEM VT)

5. DAGNELIE P., 1975. Analyse statistique à plusieurs variables. Gembloux, Belgique, Presses Agronomiques de Gembloux, $362 \mathrm{p}$.

6. FRAYSSE J.L., DARRE A., 1990. Produire des viandes, vol. 1 : Sur quelles bases économiques et biologiques ? Paris, France, Technique et documentation Lavoisier, 374 p. (Coll. agriculture d'aujourd'hui)

7. GRIMAUD P., CHARDONNET P., 1989. Comportement alimentaire du cerf rusa en croissance recevant une ration à base de graminée (Brachiaria mutica). In : Actes XVIe congrès international des herbages, 4-11 octobre 1989, Nice, France. Versailles, France, Association française pour la production fourragère, IN RA, p. 1281-1282.

8. LE BEL S., 1993. L'élevage du cerf rusa en Nouvelle-Calédonie. Bilan et perspectives. Nouméa, N ouvelle-Calédonie, CIRAD-EMVT, $26 p$. (Etudes et synthèses Projet élevage)

9. LE BEL S., CHARDONNET P., DULIEU D., SALAS M., 1995. Impact de la castration du daguet rusa sur les performances de croissance, d'abattage et sur la qualité biologique de la viande à 24 et 30 mois. Revue Elev. Med. vét. Pays trop., 48 : 85-93.

10. LE BEL S., DULIEU D., 1993. Rearing rusa deer in New Caledonia. A first attempt at relating feed management to the system of production. Noumea, N ew Caledonia, CIRAD-EMVT, p. 1-14. (Report)

11. LE BEL S., MOUZIN W., 1995. Assemblée générale de l'EDEC du 30 Juin 1995. Rapport technique. Nouméa, Nouvelle-Calédonie, CIRADEMVT, 15 p. (Etudes et synthèses Projet élevage)

12. LE BEL S., SALAS M., CHARD ONNET P., BIANCHI M., 1997. Rusa deer (Cervus timorensis russa) farming in $\mathrm{N}$ ew Caledonia: impact of different feed levels on herd breeding rate and performances of newborn fawns. Aust. vet. J., 75: 199-203. 
13. METEO FRANCE, 1993. Résumé climatologique annuel en NouvelleCalédonie. Nouméa, N ouvelle-Calédonie, Direction régionale de Météo France en N ouvelle-Calédonie et aux Iles W allis et Futuna, $6 \mathrm{p}$.

14. METEO FRANCE, 1994. Résumé climatologique annuel en NouvelleCalédonie. Nouméa, Nouvelle-Calédonie, Direction régionale de Météo France en Nouvelle-Calédonie et aux Iles W allis et Futuna, $6 \mathrm{p}$.

15. OCEF, 1993. Rapport d'activité de la section viande, $4^{e}$ trimestre. N ouméa, N ouvelle-Calédonie, OCEF.

16. OCEF, 1994. Rapport d'activité de la section viande, $4^{\mathrm{e}}$ trimestre. N ouméa, N ouvelle-Calédonie, OCEF.

17. SPSS, 1992. SPSS/PC+ Base System U ser's Guide, Version 5.0. Chicago, USA, Marijia J. N orusis/SPSS, $910 \mathrm{p}$.

18. TOUTAIN B., 1984. Principales caractéristiques des paturages de Nouvelles-Calédonie. Revue Elev. Méd. vét. N.C. (1) : 44-50.

\section{Summary}

Le Bel S., Salas M., Dulieu D. Deer farming systems in $\mathrm{New}$ Caledonia and effects of slaughtering result monitoring

A typology of the deer farms is presented using Bertin's graphic method. Four farm systems were distinguished mainly according to pasture size, average stocking rate and level of improvement. Of the 1053 deer involved in the 1994 slaughtering campaign, 927 were monitored in the survey. Most of slaughtered deer were entire stags (93\%). Thirty-five percent of the monitored population were 1 to 2 years old and $48 \%$ over 2 years old. Breeders who chose to weigh deer before slaughtering obtained a $1.6 \%$ downgraded carcass rate, whereas other breeders' was seven times higher $(12 \%$; significant difference $p<0,001$ ). The mean live weight (LW) of slaughtered deer $(n=510)$ was $49.0 \pm 0.6 \mathrm{~kg}$, the mean hot carcass weight $(\mathrm{CW})(\mathrm{n}=927)$ after drying $28.4 \pm 0.3 \mathrm{~kg}$ and the mean hot carcass dressing yield (DY) $56.8 \pm 0.3 \%$. Variance analyses on LW, CW and DY variables show that the farm system, age and season of slaughtering had a significant effect. A better performing farm could not be singled out by the farm system effect. The extensive breeding system (system 4) had lower results due to an insufficient finishing period. From 1 to 2 years old, CW increased by $6 \mathrm{~kg}$ $(25 \mathrm{~kg} / 31 \mathrm{~kg}$ ) but DY decreased by $2 \%$, from 58 to $56 \%$. The cool season was the best slaughtering season with $30 \mathrm{~kg}$ carcasses and a $57 \%$ dressing rate. $\mathrm{CW}$ can be predicted via a significant regression equation $(p<0.001)$ from LW [CW $(\mathrm{kg})$ $=0.5 \mathrm{LW}(\mathrm{kg})+3.4]$. Slaughtering performances in $\mathrm{New}$ Caledonia were not as high as those in other rusa deer farming countries. The differences are due to rather extensive rusa deer farming practices in New Caledonia. Improvement of the production level seems dependent on a better feed strategy during the dry season and an adequate finishing period of young stags slaughtered before the age of two years.

Key words: Cervidae - Cervus timorensis russa - Rusa deer Classification - Slaughtering - Carcass - W eighing - New Caledonia.
19. TOUTAIN B., DULIEU D., 1991. Pâturages et parcours en NouvelleCalédonie. Situation et perspectives. In: IVth International Rangeland Congress, Montpellier, France, A pril 22-26, 1991. Montpellier, France, CIRAD-EMVT, p. 502-504.

20. WOODFORD K., 1991. Dry argument supports Australian Rusa farming. Deer Farmer, 79: 37-39.

21. WO ODFO RD K., 1991. Deer production in tropical and subtropical environments. In: Brown R. ed., Deer branch of the NZ Veterinary Association. Deer Branch Course N 0.8. Sydney, Australia, p. 85-93.

22. WOODFORD K., DUNNING A., 1990. Production cycles and characteristics of rusa deer in Q ueensland, Australia. In: Brown R. ed., Proc. int. Symp. Biology of Deer, Mississippi State University, USA, June 1990, p. 197-202.

Reçu le 23.4.96, accepté le 12.2.98

\section{Resumen}

Le Bel S., Salas M., Dulieu D. Le Bel S., Salas M., Dulieu D. Tipología de criaderos de ciervos en N ueva Caledonia y evaluación del efecto de un seguimiento en matadero

Una tipología de los criaderos de ciervos se realizó gracias al método gráfico de Bertin. Se pueden distinguir cuatro grandes tipos de criaderos, que se diferencian esencialmente según la superficie de los pastizales, la carga media y la intensificación de las prácticas de manejo. La campaña de matadero de 1994 totalizó 1053 cabezas, de las cuáles 927 fueron incluídas en el seguimiento. Los ciervos sacrificados fueron machos enteros (93\%). Treinta y cinco por ciento del efectivo tenía entre 1 y 2 años y $48 \%$ más de dos años. El porcentaje de carcasas descartacas fue de $1,6 \%$ entre los criadores que optaron por la medida de peso como medio de escogencia; entre el resto de los criadores, fue siete veces mayor (12\%, diferencia significativa $p<0,001$ ). El peso vivo (PV) medio de los ciervos sacrificados $(n=510$ ) fue de $49,0 \mathrm{~kg} \pm 0,6 \mathrm{~kg}$, el peso medio de la carcasa $(P C)(n=927)$ caliente después secado fue de $28,4 \pm 0,3 \mathrm{~kg}$ y el rendimiento caliente (RC) medio fue de 56,8 $\pm 0,3 \%$. Los análisis de varianza llevados a cabo sobre las variables PV, PC y RC muestran que el efecto de los factores tipo de criadero, edad y época de sacrificio fue significativo. El efecto tipo de criadero no permitió detectar un tipo de explotación con mayor rendimiento. Unicamente los criaderos extensivos (tipo 4) fueron penalizados por la falta de "término" de los animales. De 1 a 2 años, el PC aumentó $6 \mathrm{~kg}(25 \mathrm{~kg} / 31 \mathrm{~kg})$, pero el RC bajó $2 \%$, de 58 a $56 \%$. La mejor época para el sacrificio fue la estación fresca, con carcasas de $30 \mathrm{~kg}$ y un rendimiento de $57 \%$. Una ecuación de regresión significativa $(p<0,001)$ permitió predecir el PC en función del PV $[P C(k g)=0,5$ PV $(k g)+3,4]$. Los rendimientos obtenidos en Nueva Caledonia no fueron tan buenos con respecto a otros países criadores de ciervos rusa. Esta diferencia de nivel de rendimiento, asociada al modo de crianza más extensivo del ciervo rusa en Nueva Caledonia, puede modificarse mediante una mejor estrategia alimentaria durante la estación seca y el engorde final de los jóvenes machos sacrificados antes de dos años.

Palabras clave: Cervidae - Cervus timorensis russa - Venado rusa - Clasificación - Sacrificio - Canal animal - Pesada Nueva Caledonia. 